\title{
Sharp Bounds on the Number of Scattering Poles for Perturbations of the Laplacian
}

\author{
Georgi Vodev \\ Section of Mathematical Physics, Institute of Mathematics, Bulgarian Academy of Sciences, \\ BG-1113 Sofia, Bulgaria
}

Received July 8, 1991; in revised form November 12, 1991

\begin{abstract}
Sharp bounds on the number $N(r)$ of the scattering poles in the disc $|z| \leqq r$ for a large class of compactly supported perturbations (not necessarily selfadjoint) of the Laplacian in $\mathbb{R}^{n}, n \geqq 3$, odd, are obtained. In particular, in the elliptic case the estimate $N(r) \leqq C r^{n}+C$ is proved.
\end{abstract}

\section{Introduction and Statement of Results}

Using the complex scaling method, Sjöstrand and Zworski [9] have recently obtained some sharp bounds on the number of the scattering poles for a large class of selfadjoint compactly supported perturbations of the Laplacian. This method allows to characterize the scattering poles as eigenvalues of another unbounded operator (in another Hilbert space) which, however, is not selfadjoint. This in turn leads to some difficulties when one tries to study its discrete spectrum. To overcome them Sjöstrand and Zworski developed a heavy machinery based in particular on an application of the spectral calculus for selfadjoint operators.

The purpose of this work is to extend the results in [9] for a class of nonselfadjoint compactly supported perturbations of the Laplacian and in particular to give a simpler proof of the bounds obtained in [9]. Let us now introduce the notations and make our assumptions. Let $G$ be a linear unbounded closed operator in a complex Hilbert space $H$ with domain $\mathscr{D}(G)$. We suppose that there exists a $\varrho_{0}>0$ so that the Hilbert space $H$ admits the orthogonal decomposition $H=H^{\prime} \oplus L^{2}\left(\mathbb{R}^{n} \backslash B_{\varrho_{0}}\right)$, where $B_{\varrho_{0}}=\left\{x \in \mathbb{R}^{n}:|x| \leqq \varrho_{0}\right\}, n \geqq 3$ is odd. If $\chi \in C_{0}^{\infty}\left(\mathbb{R}^{n}\right)$ is equal to 1 in a neighbourhood of $B_{\varrho_{0}}$ and $u \in H$ is written as $u=u_{1}+u_{2}$ with $u_{1} \in H^{\prime}$, $u_{2} \in L^{2}\left(\mathbb{R}^{n} \backslash B_{\varrho_{0}}\right)$, we define $\chi u \in H$ by $\chi u=u_{1}+\chi u_{2}$. Similarly, if $\chi \in C_{0}^{\infty}\left(\mathbb{R}^{n}\right)$ is equal to zero in a neighbourhood of $B_{\varrho_{0}}$ and to 1 outside a compact domain in $\mathbb{R}^{n}$, we

* Partially supported by Bulgarian Scientific Fondation under grant no. MM8/91 
define $\chi u$ by $\chi u=\chi u_{2} \in L^{2}\left(\mathbb{R}^{n} \backslash B_{\varrho_{0}}\right)$. We make the following assumptions:

For any $\chi \in C^{\infty}\left(\mathbb{R}^{n}\right)$ equal to zero in a neighbourhood of $B_{\varrho_{0}}$ and to 1 outside a compact domain in $\mathbb{R}^{n}$ and any $u \in \mathscr{D}(G)$, we have $\chi u \in \mathscr{D}(G) \cap H^{2}\left(\mathbb{R}^{n} \backslash B_{\varrho_{0}}\right)$ and $G \chi u=-\Delta \chi u, \Delta$ being the Laplacian in $\mathbb{R}^{n}$.

For any $\chi_{1} \in C^{\infty}\left(\mathbb{R}^{n}\right)$ as in (1.1), any $\chi_{2} \in C_{0}^{\infty}\left(\mathbb{R}^{n}\right)$ such that $\chi_{2}=1$ in a neighbourhood of $B_{\varrho_{0}}$ and $\chi_{1}=0$ on $\operatorname{supp} \chi_{2}$ and any $u \in \mathscr{D}(G)$ we have $\chi_{1} G \chi_{2} u=0$.

Instead of selfadjointness of $G$ we require the following much weaker assumption:

The resolvent set of $G$ is not empty.

It follows easily from this assumption that there exists $z_{0} \in \mathbb{C}$ with $\operatorname{Im} z_{0}>0$ and an open neighbourhood $\Lambda \subset\{z \in \mathbb{C}: \operatorname{Im} z>0\}$ of $z_{0}$ so that the resolvent $R(z)$ $=\left(G-z^{2}\right)^{-1} \in \mathfrak{L}(H, H)$ is well defined and holomorphic in $A . \mathfrak{L}(X, Y)$ denotes the space of all linear bounded operators acting from $X$ into $Y$. To introduce the scattering poles associated to the operator $G$ we need the following assumption:

There exists a function $\chi \in C_{0}^{\infty}\left(\mathbb{R}^{n}\right)$ equal to 1 in a neighbourhood of $B_{\varrho_{0}}$ so that the operator $\chi R\left(z_{0}\right)$ is compact as an operator in $\mathfrak{L}(H, H)$.

We shall show in Sect. 3 that in fact $\chi R\left(z_{0}\right)$ is compact for any other function $\chi \in C_{0}^{\infty}\left(\mathbb{R}^{n}\right)$ equal to 1 in a neighbourhood of $B_{\varrho_{0}}$. Now fix a function $\chi \in C_{0}^{\infty}\left(\mathbb{R}^{n}\right)$ equal to 1 in a neighbourhood of $B_{e_{0}}$ and consider the cutoff resolvent $R_{\chi}(z)$ $=\chi R(z) \chi$ for $z \in \Lambda$. As shown in Sect. 3 (see also [9]), under the above assumptions, $R_{\chi}(z)$ admits a meromorphic continuation from $\Lambda$ to the entire complex plane $\mathbb{C}$. The poles of this continuation are known as scattering poles or resonances. As we shall show in Sect. 3 , the poles of $R_{\chi}(z)$, as well as their multiplicities, do not depend on the function $\chi$, provided $\chi=1$ in a neighbourhood of $B_{\varrho_{0}}$. Let $\left\{z_{j}\right\}$ be the poles of $R_{\chi}(z)$ repeated according to multiplicity and set

$$
N(r)=\#\left\{z_{j}:\left|z_{j}\right| \leqq r\right\} \text {. }
$$

When the operator $G$ is selfadjoint, it is shown in [9] that

$$
N(r) \leqq C \Phi(C r) \text { for } r \geqq 1,
$$

where $C>0$ is a constant, and $\Phi(t) \in C[1, \infty)$ is an increasing function such that $\Phi(t) \geqq t^{n}$ for $t \geqq 1$ and

$$
\left|\operatorname{tr}\left(\mathscr{E}\left(h^{2} G\right) \chi\right)\right| \leqq C_{\mathscr{E}} \Phi\left(C_{\mathscr{E}} / h\right), \quad C_{\mathscr{E}}>0,
$$

$\forall h, 0<h \ll 1, \forall \mathscr{E} \in C_{0}^{\infty}(\mathbb{R})$, with some function $\chi \in C_{0}^{\infty}\left(\mathbb{R}^{n}\right)$ equal to 1 in a neighbourhood of $B_{\varrho_{0}}$. Moreover, $\Phi(t)$ is assumed to satisfy $\Phi(\theta t) \leqq C^{\prime} \theta^{\delta} \Phi(t)$ for $0<\theta \leqq 1, \theta t \geqq 1$, with some $C^{\prime}, \delta>0$. In fact, it is easy to see that the function $\Phi(t)$ can be taken to be of the same growth as the counting function of the eigenvalues of a selfadjoint operator with compact resolvent. Indeed, take a $\varrho>\varrho_{0}$ and denote by $\widetilde{G}$ the restriction of $G$ on the Hilbert space $\tilde{H}=H^{\prime} \oplus L^{2}\left(B_{\varrho} \backslash B_{\varrho 0}\right)$ with domain

$$
\mathscr{D}(\widetilde{G})=\left\{u=u_{1}+u_{2}: u_{1} \in \mathscr{D}(G) \cap H^{\prime}, u_{2} \in H^{2}\left(B_{\varrho} \backslash B_{\varrho_{0}}\right), u_{2}\left\lceil\partial B_{\varrho}=0\right\} .\right.
$$

Clearly, $\widetilde{G}$ is selfadjoint, provided so is $G$, and by the assumption (1.4) it is easy to see that the resolvent $\left(\widetilde{G}-z_{0}^{2}\right)^{-1}$ is a compact operator. Let $\left\{\lambda_{j}\right\}$ be the eigenvalues 
of $\tilde{G}$ repeated according to multiplicity and set $\tilde{N}(\lambda)=\#\left\{\lambda_{j}:\left|\lambda_{j}\right| \leqq \lambda^{2}\right\}$. If $\widetilde{\Phi}(t) \in C[1, \infty)$ is an increasing function such that $\tilde{N}(\lambda) \leqq \widetilde{\Phi}(\lambda)$ for $\lambda \geqq 1$, then it is easy to see that (1.6) holds with $\Phi(t)=\widetilde{\Phi}(t)$. Thus, the estimate (1.5) shows that the growth of the counting function of the resonances of $G$ is at most as the growth of the counting function of the eigenvalues of $\widetilde{G}$. The advantage of such a conclusion is that for many important examples the counting function $\tilde{N}(\lambda)$ is easily estimated from above for large $\lambda$. Indeed, let $G$ be a second order differential operator of the form

$$
G=-c(x)^{-1}\left(\sum_{i, j=1}^{n} \partial_{x_{i}}\left(g_{i, j}(x) \partial_{x_{j}}\right)+\sum_{j=1}^{n} b_{j}(x) \partial_{x_{j}}+a(x)\right)
$$

in $\mathbb{R}^{n}$ or in an exterior domain with Dirichlet or Neumann boundary conditions, which in additional to the above assumptions satisfies the hypoelliptic estimates

$$
\|u\|_{s+2 \varepsilon} \leqq C_{s}\left(\|G u\|_{s}+\|u\|_{s}\right), \quad \forall s \geqq 0, \quad \forall u \in \mathscr{D}(G), \quad G u \in H^{s},
$$

with some $\varepsilon, 0<\varepsilon \leqq 1$, where \|\|$_{s}$ denotes the norm in the usual Sobolev space $H^{s}$. It is not hard to see that (1.7) implies (1.4) as well as the estimate $\widetilde{N}(\lambda) \leqq C \lambda^{n / \varepsilon}$ for $\lambda \geqq 1$. Hence, in this case (1.6) is fulfilled with $\Phi(t)=t^{n / \varepsilon}$ and by (1.5) one has

$$
N(r) \leqq C r^{n / \varepsilon}+C
$$

with some constant $C>0$. Note that in the elliptic case $(\varepsilon=1)(1.8)$ also follows from the analysis in [14]. Such a bound was first proved by Melrose [5] for the Laplacian in an exterior domain with Dirichlet or Robin boundary conditions, and by Zworski [17] for the Schrödinger operator $-\Delta+V(x)$ with a potential $V \in L_{0}^{\infty}\left(\mathbb{R}^{n}\right)$.

In the present work we obtain bounds similar to (1.5) when $G$ is not selfadjoint. As a consequence, for such operators we get (1.8), provided (1.7) is fulfilled. The first difficulty in doing such a generalization is how to replace the assumption (1.6) by a similar one making sense when $G$ is not selfadjoint. This is done as follows. Given a compact operator $A$, denote by $\mu_{j}(A)$ the characteristic values of $A$, i.e. the eigenvalues of $\left(A^{*} A\right)^{1 / 2}$, repeated according to multiplicity and ordered to form a nonincreasing sequence. The assumption analogous to (1.6) is:

There exists an increasing function $f(t) \in C[1, \infty)$ such that $0<f(t) \leqq t^{1 / n}, \forall t \geqq 1$, and $\mu_{j}\left(\chi R\left(z_{0}\right)\right) \leqq C^{2} f(j)^{-2}, \forall j$, for some function $\chi \in C_{0}^{\infty}\left(\mathbb{R}^{n}\right)$ equal to 1 in a neighbourhood of $B_{\varrho_{0}}$ and a constant $C>0$ depending on $\chi$.

We shall show in Sect. 3 that if the estimate in (1.9) holds for one function $\chi$, it holds for any other function $\chi \in C_{0}^{\infty}\left(\mathbb{R}^{n}\right)$ equal to 1 in a neighbourhood of $B_{Q_{0}}$ with a constant $C=C_{\chi}>0$.

We also need the following assumption:

There exist constants $C, \delta>0, \delta<1 / 2$, so that

$$
f(\theta t) \leqq C \theta^{\delta} f(t) \text { for } \quad \theta \geqq 1, \quad t \geqq 1 .
$$

An important example of a function $f(t)$ satisfying the above assumptions is $f(t)=t^{1 / p}$ with $p \geqq n$. Denote by $\varphi(t)$ the function defined by $\varphi(f(t))=t, \forall t \geqq 1$. Since $f(t)$ is a continuous increasing function, $\varphi(t)$ is a well defined continuous increasing function, too. Moreover, it is easy to see that $f(t) \leqq t^{1 / n}$ implies $\varphi(t) \geqq t^{n}$. When $f(t)=t^{1 / p}$ with $p \geqq n$, we have $\varphi(t)=t^{p}$. Now we are ready to state our main result. 
Theorem 1. Assume (1.1)-(1.4),(1.9), and (1.10) fulfilled. Then the number $N(r)$ of the scattering poles associated to the operator $G$ satisfies the bound

$$
N(r) \leqq C \varphi(C r) \quad \text { for } \quad r \geqq 1
$$

with some constant $C>0$.

When $G$ is selfadjoint it is easy to see that the functions $\Phi(t)$ and $\varphi(t)$ can be taken to be equivalent for $t \gg 1$. So, the bounds obtained in [9] follows from the above theorem.

When $G$ satisfies (1.7), it is easy to see that (1.9) holds with $f(t)=t^{1 / p}$, where $p=n / \varepsilon$. Thus, as a consequence of the above theorem we have the following

Theorem 2. Let $G$ satisfy the assumptions (1.1)-(1.3) and (1.7). Then the number $N(r)$ of the scattering poles associated to the operator $G$ satisfies a bound of the form (1.8).

Note that for non-selfadjoint operators this result is new even in the elliptic case $(\varepsilon=1)$.

It is worth noticing that it may happen to have hypoelliptic differential operators satisfying (1.7) for which the corresponding counting function $N(r)$ of scattering poles satisfies much better bounds than (1.8). Examples for such operators come from the work [7], where asymptotics of the counting function of the eigenvalues of selfadjoint pseudodifferential operators (with positive principal symbol and double characteristics) on compact manifolds are obtained. Under some natural assumptions, these operators are hypoelliptic with loss of 1 derivative [i.e. they satisfy (1.7) with $\varepsilon=1 / 2$ ]. Although in [7] manifolds without boundary are considered only, the results apply to operators like $\widetilde{G}$ (which in this case is a second order differential operator on $B_{e}$ with Dirichlet boundary condition) since it is elliptic (equal to $-\Delta$ ) in a neighbourhood of the boundary $\partial B_{\varrho}$. In [7] three types of operators with respect to the behaviour of the counting function $\tilde{N}(\lambda)$ of eigenvalues are distinguished (we consider here only second order differential operators): (i) $\tilde{N}(\lambda)=O\left(\lambda^{n}\right)$; (ii) $\tilde{N}(\lambda)=O\left(\lambda^{n} \log \lambda\right)$; (iii) $\tilde{N}(\lambda)=O\left(\lambda^{p}\right)$ with some $p, n<p \leqq 2 n$. We refer to [7] for the details. Thus, by Theorem 1, in quite a lot of cases one has bounds for the counting function $N(r)$ of scattering poles much better than the bound $O\left(r^{2 n}\right)$ given by (1.8). All this can be well illustrated by the following particular example suggested to the author by Zworski (also, see [9]):

$$
G=-\left(\partial_{x_{1}}^{2}+\partial_{x_{2}}\left(1-\zeta(x)\left(1-x_{1}^{2}\right)\right) \partial_{x_{2}}+\partial_{x_{3}}^{2}\right),
$$

where $\zeta(x) \in C_{0}^{\infty}\left(\mathbb{R}^{3}\right)$ is a real-valued function such that $0 \leqq \zeta \leqq 1, \zeta=0$ for $|x| \geqq 1 / 2$, $\zeta=1$ for $|x| \leqq 1 / 3$. In this case $n=3, \varepsilon=1 / 2$, and $\widetilde{G}$ is the restriction of the operator $G$ on the ball $\bar{B}_{1}=\left\{x \in \mathbb{R}^{3}:|x| \leqq 1\right\}$ with Dirichlet boundary condition. By Theorem 2 we have $N(r)=O\left(r^{6}\right)$. However, the operator $\widetilde{G}$ turns out to be of type (ii) above, so by Theorem 1 one obtains the sharper bound $N(r)=O\left(r^{3} \log r\right)$.

The idea of the proof of $(1.11)$ is to find an entire family $K(z)$ of compact operators so that the poles of $R_{\chi}(z)$, with multiplicity, are among the poles of $(1-K(z))^{-1}$ and given any $r \gg 1, K(z)$ admits the decomposition $K(z)$ $=K_{r}(z)+\tilde{K}_{r}(z)$, where $K_{r}(z)$ is an entire family of trace class operators and $\left\|\tilde{K}_{r}(z)\right\|$ $\leqq 1 / 2$ for $|z| \leqq r$. Thus we obtain that the scattering poles of $R_{\chi}(z)$ in $|z| \leqq r$, with multiplicity, are among the poles of $\left(1-\left(1-\widetilde{K}_{r}(z)\right)^{-1} K_{r}(z)\right)^{-1}$, and hence among the zeros of the function

$$
h_{r}(z)=\operatorname{det}\left(1-\left(1-\tilde{K}_{r}(z)\right)^{-1} K_{r}(z)\right),
$$


which is well defined and holomorphic in $|z| \leqq r$. Now, according to Jensen's inequality, to prove (1.11) it suffices to show that

$$
\left|h_{r}(z)\right| \leqq \exp (C \varphi(C r)) \text { for }|z| \leqq r .
$$

\section{Preliminaries}

Denote by $G_{0}$ the selfadjoint realization of the Laplacian $-\Delta$ in the Hilbert space $H_{0}=L^{2}\left(\mathbb{R}^{n}\right)$ and let $R_{0}(z)$ denote the outgoing resolvent of $G_{0}$ which is by definition the operator with kernel $E(x-y ; z)$, where $E(x ; z)$ is the outgoing fundamental solution of the operator $-\Delta-z^{2}$. As is well known, the kernel of $R_{0}(z)$ is given in terms of Hankel's functions by

$$
R_{0}(z)(x, y)=(i / 4)(z / 2 \pi|x-y|)^{(n-2) / 2} H_{(n-2) / 2}^{(1)}(z|x-y|) .
$$

It is easy to see that $R_{0}(z)=\left(G_{0}-z^{2}\right)^{-1} \in \mathfrak{L}\left(H_{0}, H_{0}\right)$ for $\operatorname{Im} z>0$. Moreover, if $Q_{1}$ and $Q_{2}$ are differential operators of orders $p_{1}$ and $p_{2}$, respectively, with coefficients of class $C_{0}^{\infty}\left(\mathbb{R}^{n}\right)$, then $Q_{1} R_{0}(z) Q_{2}$ forms an entire family of pseudodifferential operators of order $p_{1}+p_{2}-2$. If we additionally assume that

$$
\operatorname{supp} Q_{1} \cap \operatorname{supp} Q_{2}=\emptyset,
$$

then $Q_{1} R_{0}(z) Q_{2}$ takes values in the pseudodifferential operators of order $-\infty$. Here $\operatorname{supp} Q_{j}$ denotes the union of the supports of the coefficients of $Q_{j}$.

Let now $Q_{1}$ and $Q_{2}$ be differential operators of orders $p_{1}$ and $p_{2}$, respectively, with coefficients of class $C_{0}^{\infty}\left(\mathbb{R}^{n}\right)$ so that $0 \leqq p_{1}+p_{2} \leqq 2$. Then, the following estimates are well known:

$$
\begin{gathered}
\left\|Q_{1} R_{0}(z) Q_{2}\right\| \leqq \exp (C\langle z\rangle), \quad \forall z \in \mathbb{C}, \\
\left\|Q_{1} R_{0}(z) Q_{2}\right\| \leqq C\langle z\rangle^{p_{1}+p_{2}-1} \quad \text { for } \quad \operatorname{Im} z \geqq 0,
\end{gathered}
$$

where \|\| denotes the norm in $\mathfrak{L}\left(H_{0}, H_{0}\right)$ and $\langle z\rangle=\left(|z|^{2}+1\right)^{1 / 2}$.

It is not hard to see that the above estimates remain valid for operators $Q_{1}$ and $Q_{2}$ of greater orders $p_{1}$ and $p_{2}$ if they satisfy (2.2). Using this, for such operators we shall show that

$$
\mu_{j}\left(Q_{1} R_{0}(z) Q_{2}\right) \leqq C_{m}\langle z\rangle^{2 m+p_{1}+p_{2}-1} j^{-2 m / n}, \quad \forall j, \quad \operatorname{Im} z \geqq 0,
$$

for any integer $m \geqq 1$ with a constant $C_{m}>0$ independent of $z$ and $j$. Before doing this, let us recall the following well known inequalities:

$$
\begin{gathered}
\left\{\mu_{j}(A B), \mu_{j}(B A)\right\} \leqq \mu_{j}(A)\|B\|, \quad \forall j, \\
\mu_{j}(A+B) \leqq \mu_{j_{2}}(A)+\mu_{j_{2}}(B), \quad \forall j,
\end{gathered}
$$

where $j_{2} \sim[j / 2]$, [a] being the integer part of $a$.

Let $\Omega$ be a ball in $\mathbb{R}^{n}$ such that $\operatorname{supp} Q_{1} \subset \Omega$ and denote by $\Delta_{\Omega}$ the selfadjoint realization of the Laplacian in the Hilbert space $L^{2}(\Omega)$ with Dirichlet boundary condition. In view of (2.6), we have

$$
\mu_{j}\left(Q_{1} R_{0}(z) Q_{2}\right) \leqq \mu_{j}\left(\left(1-\Delta_{\Omega}\right)^{-m}\right)\left\|(1-\Delta)^{m} Q_{1} R_{0}(z) Q_{2}\right\|
$$

for any integer $m \geqq 1$. Since $(1-\Delta)^{m} Q_{1}$ is a differential operator of order $2 m+p_{1}$ with coefficients of class $C_{0}^{\infty}\left(\mathbb{R}^{n}\right)$ whose support does not intersect $\operatorname{supp} Q_{2},(2.5)$ follows from the above estimate, (2.4) and the following well known estimate:

$$
\mu_{j}\left(\left(1-\Delta_{\Omega}\right)^{-m}\right) \leqq C_{m} j^{-2 m / n}, \quad \forall j .
$$


by 1 . Fix a function $\chi \in C_{0}^{\infty}\left(\mathbb{R}^{n}\right)$ equal to 1 in a neighbourhood of $B_{\varrho_{0}}$. We shall carry out the meromorphic continuation of $R_{\chi}(z)$ in a way similar to that one in [9]. Clearly, for any $z \in \mathbb{C}$ we have

$$
1=\left(G-z^{2}\right) R\left(z_{0}\right)+\left(z^{2}-z_{0}^{2}\right) R\left(z_{0}\right) .
$$

Choose functions $\chi_{1}, \chi_{2} \in C_{0}^{\infty}\left(\mathbb{R}^{n}\right)$ such that $\chi_{1}=1$ in a neighbourhood of $B_{\varrho_{0}}, \chi_{2}=1$ on $\operatorname{supp} \chi_{1}$ and $\chi=1$ on $\operatorname{supp} \chi_{2}$. Set $Q(z)=\left(1-\chi_{1}\right) R_{0}(z)\left(1-\chi_{2}\right)$ for $\operatorname{Im} z>0$. In view of the assumption (1.1) we have

$$
\left(G-z^{2}\right) Q(z)=\left(-\Delta-z^{2}\right) Q(z)=1-\chi_{2}-Q_{1} R_{0}(z)\left(1-\chi_{2}\right)
$$

for $\operatorname{Im} z>0$, where $Q_{1}=\left[\chi_{1}, \Delta\right]$. Combining (3.6) and (3.7) yields

$$
\begin{aligned}
1= & \left(G-z^{2}\right)\left(R\left(z_{0}\right)+\left(z^{2}-z_{0}^{2}\right) Q(z) R\left(z_{0}\right)\right) \\
& +\left(z^{2}-z_{0}^{2}\right)\left(Q_{1} R_{0}(z)\left(1-\chi_{2}\right) R\left(z_{0}\right)+\chi_{2} R\left(z_{0}\right)\right)
\end{aligned}
$$

for $\operatorname{Im} z>0$, which in turn implies

$$
\begin{aligned}
& R(z)\left(1-\left(z^{2}-z_{0}^{2}\right)\left(Q_{1} R_{0}(z)\left(1-\chi_{2}\right) R\left(z_{0}\right)+\chi_{2} R\left(z_{0}\right)\right)\right) \\
& \quad=R\left(z_{0}\right)+\left(z^{2}-z_{0}^{2}\right) Q(z) R\left(z_{0}\right)
\end{aligned}
$$

for $z \in \Lambda$. Multiplying both sides of this identity by $\chi$, since $Q_{1}=\chi Q_{1}$ and $\chi_{2}=\chi \chi_{2}$, we get

$$
R_{\chi}(z)(1-K(z))=R_{\chi}\left(z_{0}\right)+K_{1}(z), \text { for } z \in \Lambda,
$$

where

$$
\begin{gathered}
K(z)=\left(z^{2}-z_{0}^{2}\right)\left(Q_{1} R_{0}(z)\left(1-\chi_{2}\right) R\left(z_{0}\right) \chi+\chi_{2} R\left(z_{0}\right) \chi\right), \\
K_{1}(z)=\left(z^{2}-z_{0}^{2}\right)\left(1-\chi_{1}\right) \chi R_{0}(z)\left(1-\chi_{2}\right) R\left(z_{0}\right) \chi .
\end{gathered}
$$

By (3.1) applied with $\tilde{\chi}_{1}$ replaced by $\chi_{2}$, we have

$$
\left(1-\chi_{2}\right) R\left(z_{0}\right) \chi=R_{0}\left(z_{0}\right)\left(1-\chi_{2}\right) \chi+R_{0}\left(z_{0}\right) Q_{2} R\left(z_{0}\right) \chi,
$$

where $Q_{2}=\left[\chi_{2}, \Delta\right]$. Using this we can write the operators $K(z)$ and $K_{1}(z)$ in the form

$$
\begin{aligned}
K(z) & =K_{2}(z) K_{3}+\left(z^{2}-z_{0}^{2}\right) \chi_{2} A \chi, \\
K_{1}(z) & =K_{4}(z) K_{3},
\end{aligned}
$$

for $z \in \Lambda$, where

$$
\begin{gathered}
K_{2}(z)=Q_{1} R_{0}(z) \eta-Q_{1} R_{0}\left(z_{0}\right) \eta, \\
K_{3}=\left(1-\chi_{2}\right) \chi+Q_{2} R\left(z_{0}\right) \chi, \\
K_{4}(z)=\left(1-\chi_{1}\right)\left(\chi R_{0}(z) \eta-\chi R_{0}\left(z_{0}\right) \eta\right), \\
A=\chi R\left(z_{0}\right),
\end{gathered}
$$

$\eta \in C_{0}^{\infty}\left(\mathbb{R}^{n}\right)$ being such that $\eta=1$ on $\operatorname{supp}\left(1-\chi_{2}\right) \chi$ and $\eta=0$ on $\operatorname{supp} \chi_{1}$. Let us now see that

$$
K_{3} \in \mathfrak{L}(H, H) \text {. }
$$

Using (3.9) applied with $1-\chi_{2}$ replaced by $\eta$, we get

$$
Q_{2} R\left(z_{0}\right) \chi=Q_{2} \eta R\left(z_{0}\right) \chi=Q_{2} R_{0}\left(z_{0}\right) \eta \chi+Q_{2} R_{0}\left(z_{0}\right)[\eta, \Delta] R\left(z_{0}\right) \chi .
$$

Now this representation implies (3.11) since $Q_{2} R_{0}\left(z_{0}\right), Q_{2} R_{0}\left(z_{0}\right)[\eta, \Delta] \in \mathfrak{L}\left(H_{0}, H_{0}\right)$. 


\section{Meromorphic Continuation of the Cutoff Resolvent}

At the beginning of this section we shall show that if the assumptions (1.4) and (1.9) hold for some function $\chi_{1} \in C_{0}^{\infty}\left(\mathbb{R}^{n}\right)$ equal to 1 in a neighbourhood of $B_{00}$, they hold for any other function $\chi_{2} \in C_{0}^{\infty}\left(\mathbb{R}^{n}\right)$ with this property. Fix $\chi_{2}$ and choose a function $\chi \in C_{0}^{\infty}\left(\mathbb{R}^{n}\right)$ so that $\chi=1$ in a neighbourhood of $\operatorname{supp} \chi_{1} \cup \operatorname{supp} \chi_{2}$. Clearly, it suffices to prove the desired result with $\chi$. Choose a function $\tilde{\chi}_{1} \in C_{0}^{\infty}\left(\mathbb{R}^{n}\right)$ such that $\tilde{\chi}_{1}=1$ in a neighbourhood of $B_{e_{0}}$ and $\chi_{1}=1$ on supp $\tilde{\chi}_{1}$. In view of the assumptions (1.1) and (1.2) we have

$$
\begin{aligned}
\left(G_{0}-z_{0}^{2}\right)\left(1-\tilde{\chi}_{1}\right) R\left(z_{0}\right) & =\left(1-\tilde{\chi}_{1}\right)\left(G-z_{0}^{2}\right) R\left(z_{0}\right)+\left[\Delta, \tilde{\chi}_{1}\right] R\left(z_{0}\right) \\
& =1-\tilde{\chi}_{1}+Q R\left(z_{0}\right),
\end{aligned}
$$

where $Q=\left[\Delta, \tilde{\chi}_{1}\right]$ is the commutator of $\Delta$ and $\tilde{\chi}_{1}$. By (3.1) we easily obtain

$$
R\left(z_{0}\right)=\tilde{\chi}_{1} \chi_{1} R\left(z_{0}\right)+R_{0}\left(z_{0}\right)\left(1-\tilde{\chi}_{1}\right)+R_{0}\left(z_{0}\right) Q \chi_{1} R\left(z_{0}\right) .
$$

Multiplying (3.2) by $\chi$ on the left, we get

$$
\chi R\left(z_{0}\right)=\tilde{\chi}_{1} \chi_{1} R\left(z_{0}\right)+\chi R_{0}\left(z_{0}\right)\left(1-\tilde{\chi}_{1}\right)+\chi R_{0}\left(z_{0}\right) Q \chi_{1} R\left(z_{0}\right) .
$$

Now, since $\chi R_{0}\left(z_{0}\right)$ is a compact operator and $\chi R_{0}\left(z_{0}\right) Q \in \mathfrak{L}\left(H_{0}, H_{0}\right)$, by (3.3) we conclude that $\chi R\left(z_{0}\right)$ is a compact operator, provided so is $\chi_{1} R\left(z_{0}\right)$. Moreover, by (3.3) combined with (2.6) and (2.7), we have

$$
\mu_{j}\left(\chi R\left(z_{0}\right)\right) \leqq C\left(\mu_{j_{2}}\left(\chi_{1} R\left(z_{0}\right)\right)+\mu_{j_{2}}\left(\chi R_{0}\left(z_{0}\right)\right)\right) .
$$

On the other hand, it is well known that

$$
\mu_{j}\left(\chi R_{0}\left(z_{0}\right)\right) \leqq C_{\chi} j^{-2 / n}, \quad \forall j,
$$

This together with (3.4) and (1.10) yield

$$
\mu_{j}\left(\chi R\left(z_{0}\right)\right) \leqq C^{\prime}\left(f(j)^{-2}+j^{-2 / n}\right) \leqq 2 C^{\prime} f(j)^{-2}, \quad \forall j,
$$

provided (1.9) is fulfilled with $\chi_{1}$, which establishes (1.9) for $\chi$. Thus we have proved the independence of the assumptions (1.4) and (1.9) on the choice of the cutoff function $\chi$.

Now we shall show that the resonances, with their multiplicities, do not depend on the choice of $\chi$, provided $\chi=1$ in a neighbourhood of $B_{\varrho_{0}}$. To this end, assume that $R_{\chi_{1}}(z)$ admits a meromorphic continuation from $\Lambda$ to the entire $\mathbb{C}$ for some function $\chi_{1} \in C_{0}^{\infty}\left(\mathbb{R}^{n}\right)$ equal to 1 in a neighbourhood of $B_{\varrho_{0}}$. Let $\tilde{\chi}_{1}$ and $\chi$ be as above and make use of (3.3) with $z_{0}$ replaced by an arbitrary $z \in \Lambda$. Multiplying (3.3) by $\chi$ on the right we get

$$
R_{\chi}(z)=\tilde{\chi}_{1} R_{\chi_{1}}(z)+\chi R_{0}(z) \chi\left(1-\tilde{\chi}_{1}\right)+\chi R_{0}(z) Q R_{\chi_{1}}(z) \chi
$$

for any $z \in \Lambda$. Since $\chi R_{0}(z) \chi$ and $\chi R_{0}(z) Q$ form entire families with values in $\mathfrak{E}\left(H_{0}, H_{0}\right)$, by (3.5) we deduce that $R_{\chi}(z)$ admits a meromorphic continuation to the entire $\mathbb{C}$, provided so does $R_{\chi_{1}}(z)$, and the poles of this continuation, with multiplicity, are among the poles of $R_{\chi_{1}}(z)$. On the other hand, the identity $R_{\chi_{1}}(z)$ $=\chi_{1} R_{\chi}(z) \chi_{1}$ shows that the poles of $R_{\chi_{1}}(z)$, with multiplicity, are among the poles of $R_{\chi}(z)$. Hence the poles of $R_{\chi}(z)$ and $R_{\chi_{1}}(z)$, with multiplicity, coincide. Thus, we have proved the desired independence of the resonances on the cutoff function $\chi$.

Turn to obtaining the meromorphic continuation of the cutoff resolvent. In what follows all cutoff functions will be positive real-valued ones upper bounded 
As mentioned in Sect. 2, $K_{2}(z)$ and $K_{4}(z)$ form entire families of compact operators in $\mathfrak{L}(H, H)$. Thus, since by the assumption (1.4) the operator $A$ is compact, we conclude that the operators $K(z)$ and $K_{1}(z)$ extend analytically to the entire complex plane $\mathbb{C}$ with values in the compact operators in $\mathfrak{E}(H, H)$. Since $K\left(z_{0}\right)=0$, by the analytic Fredholm theorem $(1-K(z))^{-1}$ forms a meromorphic $\mathfrak{L}(H, H)$-valued function on $\mathbb{C}$. Thus, by (3.8), we obtain the desired meromorphic continuation of $R_{\chi}(z)$. Moreover, it is easy to see that the poles of $R_{\chi}(z)$, with multiplicity, are among the poles of $(1-K(z))^{-1}$.

\section{Proof of Theorem 1}

In what follows \|\| will denote the norm in $\mathfrak{S}(H, H)$. Since the operator $A$ is compact, it is well known that given any integer $k \geqq 1$ there exists an operator $A_{k}$ of rank $k-1$ so that

$$
\mu_{k}(A)=\left\|A-A_{k}\right\|
$$

and

$$
\mu_{j}\left(A_{k}\right)= \begin{cases}\mu_{j}(A) & \text { for } j \leqq k-1, \\ 0 & \text { for } j \geqq k\end{cases}
$$

(for example, see [1]). Fix a parameter $r \gg 1$ and denote by $k_{r}$ the least integer $\geqq \varphi(2 C r)$, where $C$ is the constant in the assumption (1.9). Then, by (4.1) and (1.9), we have

$$
\left\|A-A_{k_{r}}\right\| \leqq C^{2} f\left(k_{r}\right)^{-2} \leqq C^{2} f(\varphi(2 C r))^{-2}=(2 r)^{-2} .
$$

Set $T(z)=\left(z^{2}-z_{0}^{2}\right) \chi_{2}\left(A-A_{k_{r}}\right) \chi$. By (4.3), for $|z| \leqq r$, we have

$$
\|T(z)\| \leqq\left(|z|^{2}+\left|z_{0}\right|^{2}\right)\left\|A-A_{k_{r}}\right\| \leqq\left(r^{2}+\left|z_{0}\right|^{2}\right)(2 r)^{-2} \leqq 1 / 2,
$$

if $r \geqq r_{0}$, where $r_{0}>0$ is a constant depending on $\left|z_{0}\right|$ only. Hence, the operator $(1-T(z))^{-1}$ is well defined and holomorphic in $|z| \leqq r$ and

$$
\left\|(1-T(z))^{-1}\right\| \leqq 2 \text { for }|z| \leqq r .
$$

Now, in view of (3.10), we can write

$$
1-K(z)=(1-T(z))(1-S(z)) \text { for }|z| \leqq r,
$$

where

$$
S(z)=(1-T(z))^{-1}\left(K_{2}(z) K_{3}+\left(z^{2}-z_{0}^{2}\right) \chi_{2} A_{k_{r}} \chi\right) .
$$

Since $\operatorname{supp} Q_{1} \cap \operatorname{supp} \eta=\emptyset$, as mentioned in Sect. $2, K_{2}(z)$ forms an entire family of trace class operators. Hence, $S(z)$ is well defined and holomorphic in $|z| \leqq r$ with values in the trace class operators in $\mathfrak{L}(H, H)$. Thus, by $(4.5)$ we conclude that the poles of $(1-K(z))^{-1}$ in $|z| \leqq r$, with multiplicity, coincide with the poles of $(1-S(z))^{-1}$, and hence, introducing the function

$$
h_{r}(z)=\operatorname{det}(1-S(z)),
$$

defined and holomorphic in $|z| \leqq r$, we have that the scattering poles in $|z| \leqq r$, with multiplicity, are among the zeros of $h_{r}(z)$. Now (1.11) follows from Jensen's inequality and the following 
Lemma 1. There exists a constant $C>0$ independent of $r$ so that

$$
\left|h_{r}(z)\right| \leqq \exp (C \varphi(C r)) \quad \text { for } \quad|z| \leqq r .
$$

Indeed, by Jensen's inequality (see [10]) we have

$$
N\left(z_{0}, r / 2\right) \leqq C_{1} \sup _{|z| \leqq r} \log \left|h_{r}(z)\right|-C_{1} \log \left|h_{r}\left(z_{0}\right)\right|,
$$

where $N\left(z_{0}, r / 2\right)$ is the number of the zeros of $h_{r}(z)$, with multiplicity, in the disc $\left|z-z_{0}\right| \leqq r / 2, C_{1}>0$ is independent of $r$. Since $K\left(z_{0}\right)=0$, we have $S\left(z_{0}\right)=0$ and hence $h_{r}\left(z_{0}\right)=1$. Now (1.11) easily follows from (4.6) and (4.7).

Proof of Lemma 1. By (2.6), (2.7), and (4.4), for $|z| \leqq r$, we obtain

$$
\mu_{j}(S(z)) \leqq C \mu_{j_{2}}\left(K_{2}(z)\right)+C r^{2} \mu_{j_{2}}\left(A_{k_{r}}\right), \quad \forall j,
$$

with a constant $C>0$ independent of $z, r$, and $j$. In view of (2.3) and (4.4) we also have

$$
\mu_{j}(S(z)) \leqq\|S(z)\| \leqq \exp (C r), \quad \forall j, \quad|z| \leqq r,
$$

with a new constant $C>0$ independent of $j, z$, and $r$. Now, using Weyl's convexity estimate together with (4.8) and (4.9), we obtain

$$
\begin{aligned}
\left|h_{r}(z)\right| & \leqq \prod_{j=1}^{\infty}\left(1+\mu_{j}(S(z))\right) \\
& =\prod_{j=0}^{\infty}\left(1+\mu_{2 j+1}(S(z))\right)\left(1+\mu_{2 j+2}(S(z))\right) \\
& \leqq \prod_{j=0}^{\infty}\left(1+\mu_{2 j+1}(S(z))\right)^{2} \\
& \leqq\left(1+\mu_{1}(S(z))\right)^{2} \prod_{j=1}^{\infty}\left(1+C \mu_{j}\left(K_{2}(z)\right)+C r^{2} \mu_{j}\left(A_{k_{r}}\right)\right)^{2} \\
& \leqq e^{C r} \prod_{j=1}^{\infty}\left(1+C \mu_{j}\left(K_{2}(z)\right)\right)^{2}\left(1+C r^{2} \mu_{j}\left(A_{k_{r}}\right)\right)^{2} \\
& =e^{C r} \prod_{j=1}^{\infty}\left(1+C \mu_{j}\left(K_{2}(z)\right)\right)^{2} \prod_{j=1}^{\infty}\left(1+C r^{2} \mu_{j}\left(A_{k_{r}}\right)\right)^{2} \\
& =e^{C r} F_{1}(z) F_{2}, \quad \text { for }|z| \leqq r .
\end{aligned}
$$

We shall first estimate $F_{2}$. By (4.2) and (1.9), we have

$$
\begin{aligned}
F_{2} & \leqq \exp \left(\sum_{j=1}^{\infty} 2 C r^{2} \mu_{j}\left(A_{k_{r}}\right)\right) \\
& \leqq \exp \left(C_{1} r^{2} \sum_{j=1}^{\varphi(2 C r)} f(j)^{-2}\right) .
\end{aligned}
$$

On the other hand, using the assumption (1.10) we get

$$
\begin{aligned}
\sum_{j=1}^{m} f(j)^{-2} & \leqq \int_{1}^{m} f(s)^{-2} d s=\int_{1}^{m} s^{-2 \delta}\left(s^{\delta} f(s)^{-1}\right)^{2} d s \\
& \leqq C\left(m^{\delta} f(m)^{-1}\right)^{2} \int_{1}^{m} s^{-2 \delta} d s \leqq C(1-2 \delta)^{-1} m f(m)^{-2}
\end{aligned}
$$


By (4.11) and (4.12), we deduce

$$
F_{2} \leqq \exp \left(C^{\prime} \varphi\left(C^{\prime} r\right)\right)
$$

with constant $C^{\prime}>0$ independent of $r$.

Now we are going to estimate $F_{1}(z)$ for $\operatorname{Im} z \geqq 0$. By (2.4) applied with $p_{1}=1$ and $p_{2}=0$, we have

$$
\mu_{j}\left(K_{2}(z)\right) \leqq\left\|K_{2}(z)\right\| \leqq C, \quad \forall j, \quad \operatorname{Im} z \geqq 0,
$$

with a constant $C>0$ independent of $z$ and $j$. On the other hand, applying (2.5) with $m=(n+1) / 2, p_{1}=1$ and $p_{2}=0$ yields

$$
\mu_{j}\left(K_{2}(z)\right) \leqq C\langle z\rangle^{n+1} j^{-(n+1) / n}, \quad \forall j, \quad \operatorname{Im} z \geqq 0,
$$

with another constant $C>0$ independent of $z$ and $j$. Now, by (4.14) and (4.15), for $\operatorname{Im} z \geqq 0$, we have

$$
\begin{aligned}
F_{1}(z) & \leqq \prod_{j \leqq\langle z\rangle^{n}}\left(1+C \mu_{j}\left(K_{2}(z)\right)\right)^{2} \prod_{j \geqq\langle z\rangle^{n}}\left(1+C \mu_{j}\left(K_{2}(z)\right)\right)^{2} \\
& \leqq\left(\prod_{j \leqq\langle z\rangle^{n}} C^{\prime}\right) \exp \left(\sum_{j \geqq\langle z\rangle^{n}} 2 C \mu_{j}\left(K_{2}(z)\right)\right) \\
& \leqq \exp \left(C^{\prime \prime}\langle z\rangle^{n}\right) \exp \left(C^{\prime \prime}\langle z\rangle^{n+1} \sum_{j \geqq\langle z\rangle^{n}} j^{-(n+1) / n}\right)
\end{aligned}
$$

On the other hand, we have

$$
\sum_{j=s}^{\infty} j^{-(n+1) / n} \leqq \int_{s}^{\infty} j^{-(n+1) / n} d j=n s^{-1 / n} .
$$

By (4.16) and (4.17), we deduce

$$
F_{1}(z) \leqq \exp \left(C_{1}\langle z\rangle^{n}\right) \leqq \exp \left(C r^{n}\right) \text { for }|z| \leqq r, \quad \operatorname{Im} z \geqq 0,
$$

with a constant $C>0$ independent of $z$ and $r$.

It remains to estimate $F_{1}(z)$ for $\operatorname{Im} z \leqq 0,|z| \leqq r$. Then, as above, we have

$$
\begin{aligned}
F_{1}(z) & \leqq \prod_{j=0}^{\infty}\left(1+C \mu_{2 j+1}\left(K_{2}(z)\right)\right)^{4} \\
& \leqq e^{C r} \prod_{j=1}^{\infty}\left(1+C \mu_{j}\left(K_{2}(-z)\right)+C \mu_{j}\left(K_{2}(z)-K_{2}(-z)\right)\right)^{4} \\
& \leqq e^{C r} \prod_{j=1}^{\infty}\left(1+C \mu_{j}\left(K_{2}(-z)\right)\right)^{4}\left(1+C \mu_{j}\left(K_{2}(z)-K_{2}(-z)\right)\right)^{4} \\
& =e^{C r} \prod_{j=1}^{\infty}\left(1+C \mu_{j}\left(K_{2}(-z)\right)\right)^{4} \prod_{j=1}^{\infty}\left(1+C \mu_{j}(L(z))\right)^{4} \\
& =e^{C r} F_{3}(z) F_{4}(z),
\end{aligned}
$$

where $L(z)=K_{2}(z)-K_{2}(-z)=Q_{1}\left(R_{0}(z)-R_{0}(-z)\right) \eta$. We have already seen above that

$$
F_{3}(z) \leqq \exp \left(C_{1}^{\prime}\langle z\rangle^{n}\right) \leqq \exp \left(C^{\prime} r^{n}\right) \text { for }|z| \leqq r .
$$

To estimate $F_{4}(z)$, observe that it follows easily from (2.1) that the kernel of $R_{0}(z)-R_{0}(-z)$ is given by

$$
(i / 2)(2 \pi)^{-n+1} z^{n-2} \int_{\mathbb{S}^{n-1}} \exp (i z\langle x-y, w\rangle) d w, \quad x, y \in \mathbb{R}^{n},
$$


where $\mathbb{S}^{n-1}$ denotes the unit sphere in $\mathbb{R}^{n}$. Using this representation, in the same way as in [14], one can obtain that

$$
\mu_{j}(L(z)) \leqq C j^{-n /(n-1)} \quad \text { for } \quad j \geqq C\langle z\rangle^{n-1}, \quad \forall z \in \mathbb{C},
$$

with a constant $C>0$ independent of $j$ and $z$. Also, it is easy to see that

$$
\left.\mu_{j}(L(z)) \leqq\|L(z)\| \leqq \exp (C<z\rangle\right), \quad \forall j, \quad \forall z \in \mathbb{C},
$$

with a constant $C>0$ independent of $j$ and $z$. Now, using (4.21) and (4.22) we get

$$
\begin{aligned}
F_{4}(z) & \leqq \prod_{j \leqq C\langle z\rangle^{n-1}}\left(1+C \mu_{j}(L(z))\right)^{4} \exp \left(\sum_{j \geqq C\langle z\rangle^{n-1}} 4 C \mu_{j}(L(z))\right) \\
& \leqq\left(\prod_{j \leqq C\langle z\rangle^{n-1}} e^{C^{\prime}\langle z\rangle}\right) \exp \left(C^{\prime} \sum_{j=1}^{\infty} j^{-n /(n-1)}\right) \\
& \leqq \exp \left(C^{\prime \prime}\langle z\rangle^{n}\right) \leqq \exp \left(C r^{n}\right), \text { for }|z| \leqq r,
\end{aligned}
$$

with a constant $C>0$ independent of $z$ and $r$. Thus, by (4.18)-(4.20) and (4.23) we deduce

$$
F_{1}(z) \leqq \exp \left(C r^{n}\right) \text { for }|z| \leqq r,
$$

with some constant $C>0$ independent of $z$ and $r$. Now (4.6) follows from (4.10), (4.13), and (4.24) at once. This completes the proof of Lemma 1.

Acknowledgments. The author would like to thank Vesselin Petkov for the useful discussions and for his encouragement.

\section{References}

1. Gohberg, I., Krein, M.: Introduction to the theory of linear non-selfadjoint operators. Providence, RI: AMS, 1969

2. Intissar, A.: A polynomial bound on the number of scattering poles for a potential in even dimensional space $\mathbb{R}^{n}$. Commun. Partial Differ. Eqs. 11, 367-396 (1986)

3. Lax, P.D., Phillips, R.S.: Scattering theory. New York: Academic Press 1967

4. Melrose, R.B.: Polynomial bounds on the number of scattering poles. J. Funct. Anal. 53, 287-303 (1983)

5. Melrose, R.B.: Polynomial bounds on the distribution of poles in scattering by an obstacle. Journées "Equations aux Dérivées Partielle," Saint-Jean-de-Montes, 1984

6. Melrose, R.B.: Weyl asymptotics for the phase in obstacle scattering. Commun. Partial Differ. Eqs. 13, 1431-1439 (1988)

7. Menikoff, A., Sjöstrand, J.: On the eigenvalues of a class of hypoelliptic operators. Math. Ann. 235, 55-85 (1978)

8. Sjöstrand, J.: Geometric bounds on the number of resonances for semiclassical problems. Duke Math. J. 60, 1-57 (1990)

9. Sjöstrand, J., Zworski, M.: Complex scaling and distribution of scattering poles. J. Am. Math. Soc. (to appear)

10. Titchmarsh, E.C.: The theory of functions, Oxford: Oxford University Press 1968

11. Vainberg, B.: Asymptotic methods in equations of mathematical physics. New York: Gordon and Breach, 1988

12. Vodev, G.: Polynomial bounds on the number of scattering poles for symmetric systems. Ann. Inst. H. Poincaré (Physique Théorique) 54, 199-208 (1991) 
13. Vodev, G.: Polynomial bounds on the number of scattering poles for metric perturbations of the Laplacian in $\mathbb{R}^{n}, n \geqq 3$, odd. Osaka J. Math. 28, 441-449 (1991)

14. Vodev, G.: Sharp polynomial bounds on the number of scattering poles for metric perturbations of the Laplacian in $\mathbb{R}^{n}$. Math. Ann. 291, 39-49 (1991)

15. Zworski, M.: Distribution of poles for scattering on the real line. J. Funct. Anal. 73, 277-296 (1987)

16. Zworski, M.: Sharp polynomial bounds on the number of scattering poles of radial potentials. J. Funct. Anal. 82, 370-403 (1989)

17. Zworski, M.: Sharp polynomial bounds on the number of scattering poles. Duke Math. J. 59, 311-323 (1989)

Communicated by B. Simon 